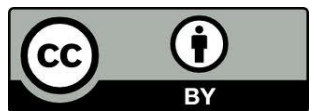

\title{
LA HERMENÉUTICA ANALÓGICA COMO ENFOQUE METODOLÓGICO PARA LA INTERPRETACIÓN DE EXPERIENCIAS
}

\author{
Analog Hermeneutics as Methodological Approach to Interpretation of Experiences
}

\author{
David Vides \\ Universidad La Salle de Bogotá \\ Doctorado en Educación \\ E-mail: demmanuel01@gmail.com
}

\begin{abstract}
RESUMEN: Como parte del proyecto de investigación La Educación Religiosa Escolar como espacio de formación política, se presenta la adaptación de algunos lineamientos de la Hermenéutica Analógica, planteada por Mauricio Beuchot. La opción por esta corriente hermenéutica pasa por el reconocimiento de que el objetivo del proyecto asume como mediación las experiencias de docentes, y ello implica un ejercicio de interpretación y elaboración de nuevos sentidos. Dos partes componen el artículo: en primer lugar, el abordaje teórico de los principales supuestos de la hermenéutica analógica; se plantean allí, elementos históricos de la hermenéutica en general, una aproximación al concepto de analogía y sus implicaciones en la interpretación, y finalmente, la pertinencia de este enfoque para el abordaje del proyecto. En segundo lugar, se exponen sus posibilidades metodológicas generales, las opciones particulares -incluyendo algunas consideraciones sobre las experiencias de los docentes como texto susceptible de interpretación analógica, y aspectos referidos a las técnicas y la conformación de la muestra-, y por último, la forma como estas opciones se tradujeron en fases de investigación y en un diseño específico.
\end{abstract}

Palavras chave: Hermenéutica Analógica, Religión, Política, Metodología, Experiencias.

ABSTRACT: As part of the research project School Religious Education as a space for political education, adaptation of some guidelines for Analog Hermeneutics, raised by Mauricio Beuchot presented. The option for this hermeneutical current passes through the recognition that the objective of the project assumed as mediation experiences of teachers, and this involves an exercise of interpretation and elaboration of new meanings. Two parts compose the article: first, the theoretical approach of the main assumptions of the analog hermeneutics; there arise, historical elements of hermeneutics in general, an approach to the concept of analogy and its implications for the interpretation, and finally, the relevance of this approach to approach the project. Second, its general methodological possibilities exposed, particular options including some considerations on the experiences of teachers as susceptible analogue text interpretation, and matters relating to technical and conformation of the sample, and finally, so how are you options resulted in research phases and in a specific design.

Keywords: Analog Hermeneutics, Religion, Politics, Methodology, Experiential. 
El objetivo del proyecto de investigación La Educación Religiosa Escolar como espacio de Formación Política, de proponer un marco antropopedagógico que permita la vinculación de la formación política al espacio de Educación Religiosa Escolar, asume la mediación de las experiencias de docentes de instituciones educativas distritales de la ciudad de Bogotá, en un ejercicio de interpretación de experiencias y elaboración conjunta de nuevos sentidos, siguiendo los lineamientos de la hermenéutica analógica, planteada por Mauricio Beuchot; se reconoce en ella un esfuerzo de equilibrio entre las posturas unívocas que llevan a fanatismos y totalitarismos en los ámbitos de referencia y las posturas equivocistas que conducen a un relativismo también inadecuado en el marco de una propuesta pedagógica o de formación.

La perspectiva metodológica se plantea con base en dos presupuestos: la importancia de la subjetividad que se enmarca en la categoría de las experiencias de los docentes, y, la concepción del proyecto en relación con su impacto social en comunidades escolares concretas. En ese sentido, es fundamental el reconocimiento de la complejidad de la realidad escolar al momento de su abordaje y comprensión, más aún desde un enfoque participativo. De hecho, la posibilidad de riesgos como la imposición de estrategias externas y mecanismos homogenizantes, conlleva la planeación misma de espacios de apropiación, de intervención en la construcción colectiva y de concienciación del tiempo natural necesario para alcanzar los objetivos propuestos. En últimas, es el reconocimiento de que un proyecto de la índole que se propone a mediano plazo tiene incidencias en los Proyectos Educativos Institucionales $y$, por tanto, debe echar raíces en la cultura escolar misma.

Teniendo eso en cuenta, el proyecto se inscribe en el paradigma cualitativo desde la perspectiva de atención a las experiencias de los sujetos con la finalidad de transformar prácticas concretas; en ese sentido, la intencionalidad se plantea desde la comprensión de la realidad a partir de las experiencias, donde entra en juego, la concepción de la investigación como oportunidad de dar voz a los sujetos y se incorpora la comunidad en la construcción misma de propuestas alternativas.

\section{La hermenéutica analógica: entre el univocismo y el equivocismo}

Beuchot (2009) define la hermenéutica como la disciplina de la interpretación de textos, y en el caso específico de la hermenéutica analógica, como aquella que hace uso del concepto de analogía para estructurarse. Teniendo esto en cuenta, inicialmente se exponen las 
consideraciones respecto al concepto de hermenéutica; y más adelante, las relacionadas con el concepto de analogía como criterio de proporcionalidad; para terminar, la relación del enfoque analógico con los ámbitos de lo religioso y lo político, como expresión de su pertinencia para el proyecto.

Recorrido histórico de la hermenéutica. Antes de que Schleiermacher la asumiera como una postura filosófica, la hermenéutica había sido considerada únicamente como una técnica de interpretación de textos. A partir de ahí inició un camino que pasa por Dilthey quien la postuló como método de las ciencias del espíritu, Heidegger quien la aplicó a la fenomenología de Husserl y planteó el círculo hermenéutico donde se incluye el condicionamiento de la interpretación por los conocimientos previos, Gadamer que basado en los planteamientos de su maestro plantea la interpretación desde una tradición pero impone la obligación de innovar y la posibilidad de cierto grado de objetividad en consciencia de los prejuicios propios, y, Ricoeur quien inició en la fenomenología pero frente a circunstancias como la voluntad y el problema del mal, que solo se expresan a través de mitos y símbolos, identificó la necesidad de la interpretación. En la perspectiva de Gadamer, se ha logrado cierta continuidad desde perspectivas distintas: por una parte Richard Rorty y Gianni Vattimo, quienes han llegado a posturas extremas de negación de toda verdad y objetividad, y por otra, Charles Taylor y Jean Grondin, que presentan la importancia de cierto equilibrio.

Mirando más de cerca estas vertientes, se plantea que si bien "la hermenéutica se ha posicionado en la filosofía contemporánea" (Beuchot, 2012, p. 184), se advierte la división entre las hermenéuticas univocistas, herederas del legado positivista de la modernidad, y las equivocistas, propias de la posmodernidad llegando incluso a un relativismo extremo; frente a esta dualidad, que evidencia la tensión en la que se mueve el pensamiento actual, se plantea la necesidad de una hermenéutica intermediaria y mediadora. En las hermenéuticas equivocistas, se incluye a Vattimo quien plantea que frente al totalitarismo de los univocismos, "solamente una hermenéutica equívoca puede garantizar que todos hagamos lo que nos parece correcto en la sociedad" (p. 187); sin embargo, considera esta postura como un relativismo extremo cuyos límites necesarios pueden ser dados por la analogía. Respecto a las hermenéuticas univocistas, se plantea la perspectiva de Jean Grondin quien afirma que "todos buscamos en el fondo, la univocidad, al perseguir $L a$ interpretación; esto es, la interpretación verdadera y única" (p. 187).

De hecho, el pensamiento actual evidencia la tensión entre la filosofía analítica y la posmoderna. La filosofía analítica es típicamente moderna especialmente en el neopositivismo, que recupera elementos como el rigor, la objetividad, centrándose en la lógica formal, la filosofía del lenguaje y la de la ciencia; actualmente se mueve entre el positivismo lógico y el pragmatismo, que es su vertiente más cercana al movimiento posmoderno; no obstante, el 
rigorismo positivista continúa estando presente incluso ante problemas como los de la antropología filosófica en la que se da más peso al discurso biológico que al cultural. En últimas, se evidencia una postura univocista cuyo principal rasgo es el apego a la objetividad reduccionista. Por su parte, la filosofía posmoderna se edifica en buena parte, en su oposición a la modernidad y la crítica a sus valores, entre los cuales prevalecen la verdad y el valor absolutos; de esta manera, ha implicado una orientación hacia la hermenéutica como instrumento conceptual, constituyéndose en la característica de su pensamiento. Con todo, es posible afirmar que ha encontrado conexión con posturas que van desde la filosofía analítica y el pragmatismo hasta posturas más subjetivistas y relativistas.

Ahora bien, entre los planteamientos posmodernos y la cercanía con la filosofía analítica, aparece el denominado camino analogista en Gadamer y Ricoeur. De Gadamer, se rescata la recuperación de elementos aristotélicos entre los cuales destacan las virtudes de la phrónesis o prudencia y la justicia; de hecho, la interpretación como acción situada, contextual, no abstracta, remitiría a la phrónesis en cuanto proporción; en otras palabras, la phrónesis se constituiría en modelo de la hermenéutica en cuanto búsqueda de proporción, equiparándola con la analogía en el hecho de dar a cada cosa la porción que le corresponde; en el caso de los textos, un significado proporcional. A su vez, la justicia se entendería desde la proporción. En esta línea, el valor del concepto de analogía se da desde la recuperación de un equilibrio proporcionado necesario en la vida social; "es una manera de privilegiar lo más posible la diferencia, sin perder la capacidad de universalizar. Es decir, nos ayuda a buscar lo propio de cada conjunto sin olvidar su común denominador" (p. 188-189).

Por su parte, la noción de analogía se halla muy presente también en Ricoeur. Primero, por su idea del símbolo, que siendo lo más propio de una cultura, sólo se puede interpretar por analogía, para evitar caer en una significación cerrada ni diluirlo entre infinitos significados. Segundo, por su consideración de la justicia que, desde esta perspectiva, aparece plenamente analógica, en la proporción -desde la justicia conmutativa y legal- y la atribución -desde la justicia distributiva-. Las implicaciones en el ámbito social y político son evidentes, especialmente en una sociedad en la que se establecen los derechos humanos. A continuación, las implicaciones en el concepto de analogía.

La noción de analogía y sus implicaciones en la interpretación. Si se ha definido la hermenéutica como la disciplina de la interpretación de textos, la opción por el concepto de analogía implica que "la hermenéutica analógica pretende estructurar la interpretación con el esquema de la analogía, lo cual es un modo de significar que se coloca entre la univocidad y la equivocidad" (Beuchot, 2008, p. 495). La explicación de este concepto se basa en la filosofía del lenguaje y la semántica; se encuentran allí, tres modos de significar: la univocidad que se 
puede definir como la significación idéntica de un término con respecto a sus significados, la equivocidad que constituye la significación completamente diferente de un término con respecto a sus significados, y la analogía como significación, en parte idéntica, en parte diferente, predominando la diferencia; en otras palabras, el modo de significación analógico, oscila entre los anteriores, múltiples significaciones pero relacionadas entre sí, con una jerarquía y orden. Al aplicar estos conceptos a la hermenéutica nos encontramos: primero, con una interpretación unívoca, que pretende ser la única objetiva o verdadera, segundo, con una interpretación equívoca, que no aspira a la verdad ni a la objetividad pues todas las interpretaciones son posibles y válidas, y finalmente, con una interpretación analógica que trata de ser abierta pero con exigencia de rigor, esto es, la aceptación de un conjunto de interpretaciones cuya validez se establece en un orden jerárquico desde un analogado principal a unos secundarios, en orden decreciente hasta el punto en que comienzan a considerarse falsas las interpretaciones.

Por otra parte, existen dos tipos de analogía: la de atribución y la de proporcionalidad. La primera, de origen pitagórico, señala el común denominador de los elementos, y la segunda, de origen platónico, señala los grados de adecuación de un atributo con respecto a varios sujetos; de esta última es de donde se recupera el orden jerárquico de los analogados, principal y secundarios. A su vez, la analogía de proporcionalidad se divide en propia o literal e impropia o metafórica, que son expresión de las dos caras de la analogía: la metonimia y la metáfora; la metonimia se da en la analogía de atribución y en la de proporcionalidad propia, y la metáfora se da en la analogía de proporcionalidad impropia. Al ser tanto la metonimia como la metáfora abarcadas por la analogía, más que una oposición entre hermenéuticas metonímicas versus hermenéuticas metafóricas, se plantea una hermenéutica estructurada en la analogía que permitiera el abordaje de ambos tipos de discursos. Del mismo, modo, las nociones de metáfora y metonimia se unen en el signo icónico, que es el análogo; la iconicidad permite pasar del efecto a la causa y de la parte al todo, pero en una explicación y universalización cargada de fuerza metafórica.

Planteado lo anterior, la pregunta ¿qué es interpretar analógicamente?, se responde en tres sentidos: en primer lugar, es interpretar un texto buscando una coherencia interna, proporcional entre sus elementos constitutivos; la sintaxis como orden, coordinación. En segundo lugar, es interpretar buscando la relación proporcional del texto con los objetos o hechos que designa; la semántica como referencia al mundo. En tercer lugar, es interpretar buscando proporcionalmente el uso del autor, su intencionalidad expresiva y comunicativa; la pragmática como lectura del intérprete proporcional a la escritura del autor. En otras palabras, en la analogía la palabra clave es la proporcionalidad, y esto se traduce en la búsqueda de un orden de coherencia proporcional, una correspondencia proporcional entre el texto y el mundo 
del texto, y, una relación proporcional entre la intencionalidad del autor y la del lector. Existe entonces una consciencia de que si bien, no se puede alcanzar el sentido literal, la referencia rígida y la intencionalidad plena del autor -todas ellas expresiones univocistas-, tampoco es posible renunciar so riesgo de caer en un sentido puramente alegórico, sin referencialidad alguna y aludiendo únicamente a la intencionalidad del lector -todas ellas expresiones equivocistas:

En una hermenéutica analógica se trata de abrir el abanico de las interpretaciones, más allá de la hermenéutica unívoca, que es muy rígida, y considerar varias interpretaciones como válidas (no sólo una), pero a diferencia de la hermenéutica equívoca, un conjunto finito y ordenado de ellas (no infinito ni homogéneo), en el que se pueda distinguir cuáles son mejores que otras, con parámetros no rígidos, pero serios, como el haber trabajado en las condiciones del lenguaje, haber estudiado la época del autor, para tener el contexto adecuado (Beuchot, 2010, p. 39).

De tal forma, una hermenéutica analógica evitaría los inconvenientes de los dos extremos; si el concepto analógico se mueve entre la univocidad y la equivocidad, esto supone un término medio entre la pretensión de claridad y distinción, y la caída en la oscuridad y confusión. Esta coincidencia de opuestos, que no sólo se dejan coexistir sino que se colaboran mutuamente, tiene varias implicaciones: busca el equilibrio proporcional entre el sentido literal y el alegórico de los textos; permite oscilar entre la metonimia y la metáfora, según el carácter del texto; y, da pie a un punto medio entre una lectura sintagmática -horizontal y superficial- y paradigmática -vertical y en profundidad-. Cabe decir que la oscilación entre ambos extremos, ha dado pie a plantear cierta dialéctica en la analogía que Scanonne ha llamado "analéctica" (p. 41); a diferencia de la dialéctica hegeliana, la analéctica no llega a la síntesis de los opuestos, sino a una conciliación frágil constituyéndose en una dialéctica de la diferencia.

Pertinencia de la hermenéutica analógica. Teniendo en cuenta lo anterior, la pertinencia de la hermenéutica analógica, pasa por la necesidad de una comprensión que supere los dualismos -entre lo físico y lo espiritual, lo público y lo privado, entre otrosy permita una aproximación a la diversidad que en el caso del proyecto se traduce en la comprensión de las categorías propias en términos de univocidad, equivocidad y analogía. En esa perspectiva, la apuesta por lo analógico parte de la complejidad del ser humano y sus situaciones propias; ante la necesidad de partir de una fundamentación antropológica, es indispensable el reconocimiento de que entre una comprensión de ser 
humano unívoca - una única comprensión de ser humano que serviría para los fines propuestos- y la aceptación de comprensiones equívocas -cualquier concepción de ser humano-, la alternativa de una comprensión analógica da espacio a la diversidad y a una visión alternativa acorde con un espacio de Educación Religiosa que apueste por la apertura desde los fundamentos mismos del ser humano. En palabras de Alvarez (2009): "La interpretación analógica lejos de ser un modo particular de conocimiento, más o menos secundario y añadido, es precisamente lo que caracteriza al ser humano como tal, su peculiar modo de ser" (p. 244).

Una de las razones que permiten evidenciar la pertinencia de la hermenéutica analógica al ámbito religioso es la correspondencia del univocismo y equivocismo con posturas frecuentes frente a la diversidad religiosa: la validez de una sola creencia como válida en un menosprecio o subvaloración de otras, o bien, la aceptación de validez de toda creencia, sin importar sus implicaciones sociales y políticas. En este caso, lo confesional puede convertirse en el elemento que remite a lo trascendente en el ser humano -aquello de lo cual no puede sustraerse: el sufrimiento, la muerte, la esperanza-, y desde allí generar un criterio desde el cual iniciar ciertos analogados. A continuación, algunos elementos que relacionan la hermenéutica analógica con las categorías centrales.

Hermenéutica analógica y Religión. La relación de la hermenéutica analógica con la religión, pasa por la consideración de la filosofía de la religión en su objetivo de buscar el sentido del fenómeno religioso en las circunstancias contemporáneas. Entre la filosofía analítica y sus exigencias de referencia y demostración y la filosofía posmoderna, que se mueve en las búsquedas de sentido desde los contextos, la perspectiva analógica permite la unión de sentido y referencia, en torno al fenómeno religioso.

En efecto, el estudio del fenómeno religioso, también se encuentra en medio de la polarización entre la interpretación reduccionista, unívoca, y la interpretación irreductible, equívoca, ante las cuales se posiciona la interpretación analógica, como interpretación que procura ser abierta pero con límite. En primer lugar, la filosofía analítica tiene toda una filosofía de la religión consolidada, que aborda el tema de lo religioso en su aspecto lógico, lingüístico y epistemológico; de alguna forma, se preocupa por la cientificidad del conocimiento religioso y en ello aborda el problema 
del lenguaje sobre Dios. Si bien, ha renunciado al verificacionismo que se aplicaba incluso a la existencia de Dios, -desde pruebas deductivas que pudieran constituirse en demostración de algo más allá de lo físico-, parece ocuparse ahora del falsacionismo, siguiendo los postulados de Popper; en esa lógica, la religión como saber, al no contar con criterios de falsación no podía aspirar a la cientificidad, pero tampoco quedaba invalidada como falsa. Con ello, aparecen posturas como las del pragmatismo que plantea que se puede adoptar la religión de acuerdo con su provecho para nuestra existencia; sin embargo, parece ser el mayor grado de apertura que es posible encontrar en esta corriente; de hecho, partiendo de la invocación que hacen las religiones, de seres que no son susceptibles de nuestros criterios de existencia, no sería posible la participación de líderes religiosos en el diálogo socio-político propuesto por Habermas, por ejemplo.

Frente a esta epistemología objetivista, empirista y reduccionista, la religión ha suscitado interés para la filosofía posmoderna, sea para asumir un pensamiento explícitamente religioso, o bien, para reconocer la dimensión religiosa como parte integrante del ser humano que habría que afrontar filosóficamente. Como se ha planteado, en este enfoque, se corre el riesgo del relativismo; la teología resultante resalta la diferencia y la otredad, hasta caer en un subjetivismo, que establece relaciones con el multiculturalismo y otros temas relacionados con la diferencia y la identidad. Beuchot plantea el pensamiento de Jean-Luc Marion como ejemplo de esta corriente posmoderna, que después de desechar toda metafísica ha ido recuperándola a través de la noción de analogía. Son de rescatar dos de sus postulados fundamentales: el fenómeno saturado y la hermenéutica del ícono; el primero, es aquel "que tiene de suyo una gran evidencia, pero en cuanto a nosotros se oculta. Un ejemplo de esto es el rostro del otro, al cual Marion llama ícono" (p. 34). Para este ícono establece una hermenéutica infinita pero que debe encontrar un límite, y es el de la propia finitud; entre la univocidad que no se puede alcanzar en el fenómeno saturado y la equivocidad de la infinitud desordenada, se plantea la propuesta de Marion como analógica.

El tipo de respuesta religiosa que originan estas dos corrientes podría definirse desde los componentes de la semiótica a los que se hizo alusión inicialmente, afirmando que la filosofía analítica está más orientada hacia la referencia de lo religioso mientras que la filosofía posmoderna se orienta más hacia el sentido. En esta línea, la filosofía 
analítica se preocupa más por la semántica del discurso religioso, esto es, si designa algo en la realidad, a partir de parámetros empíricos que permitan un estatuto epistemológico objetivo y confiable; este conocimiento de lo trascendente, configurado como filosofía de la religión, se asimila en algo a lo que antes se denominaba teodicea. En contraste, la filosofía posmoderna se pregunta más que por la existencia de la deidad, por el sentido que puede suscitarse desde la creencia, especialmente asumiendo el problema de la falta de sentido: "es el sentido el que se nos ha caído; estamos en un mundo de símbolos rotos" (p. 37). Cabe decir que el discurso religioso y la filosofía de la religión harían parte de los metarrelatos que la posmodernidad ha derruido, de donde surgen formas de religiosidad no como metarrelatos sino desde las particularidades culturales y personales; asimismo, la creciente importancia de lo estético, lleva a la religión a una focalización en la liturgia, el arte sacro y las teologías narrativas.

Hermenéutica analógica y Política. La hermenéutica analógica también se presenta como un instrumento conceptual que puede aplicarse a la política. Como se planteó anteriormente, la noción de analogía, entendida desde su origen griego como proporción implica elementos como la justicia y la prudencia que se asumen necesarios para la vida política; de hecho, el camino analogista ya planteado pasa por las consideraciones de Gadamer y Ricoeur al respecto. Más aún, al entender la hermenéutica como la disciplina de la interpretación de textos y en reconocimiento de los distintos tipos de textos susceptibles de ser interpretados, entre los cuales podrían contarse la interacción social y política, no es extraña la aplicación de la hermenéutica a este ámbito. Entre quienes han aplicado la hermenéutica a la política, Beuchot (2012) menciona a Niestzsche, Habermas y Foucault, en una línea crítica, y, por otra parte, John Rawls, Michael Walzer y Charles Taylor (p. 182).

Dicho esto, la aplicación de la hermenéutica analógica a la política se plantea desde la necesidad de interpretar al ser humano y cuál es su bien, de forma que sea posible la edificación de una polis y el logro del bien común; "solamente si comprendemos al ser humano le daremos la sociedad y la política que necesita. Y, de esta manera, se tratará de una interpretación que transforma la realidad" (p. 192). Así, se plantea una consideración de la filosofía política como hermenéutica de la facticidad social, y ello, con un carácter dinámico desde la dialéctica, a partir de la consideración del devenir que se expresa en el conflicto y las interacciones humanas. La aplicación de 
la hermenéutica analógica a la filosofía política permite evitar una comprensión unilateral y empobrecida del ser humano, -unívoca en su reduccionismo-, así como, la atomización equívoca del mismo en un relativismo desmedido; sin la pretensión de una exactitud perfecta, la búsqueda se encaminaría en el rigor aproximado de una visión analógica de la sociedad y sus problemáticas, panorama visiblemente correspondiente con los alcances reales de las ciencias sociales y humanidades. Dicho esto, no como renuncia, resignación, sino en reconocimiento de un carácter epistemológico propio más cercano a la analogía que al status unívoco de las ciencias exactas, y cuyo influjo ha llevado a la adquisición de un ropaje formalista y matemático, en ocasiones no adecuado. Sobra decir, que la comprensión analógica también evitaría la laxitud extrema en metodologías, para perseguir un punto medio, el "equilibrio proporcional que nos da la analogía, el conocimiento analógico, que es el de lo vivo, el de lo dinámico" (p. 193).

\section{La hermenéutica analógica como metodología}

Entre los planteamientos sobre la amplitud del carácter metodológico de la hermenéutica y las consideraciones de que hay categorías que se constituyen en una condición de acción para el acto interpretativo, la hermenéutica analógica plantea ciertos elementos fundamentales cuyo rigor, sin embargo, no debe asumirse desde la rigidez del tradicionalmente llamado método científico en aras de una pretensión de validez universal que hace parte del legado del positivismo moderno.

Para comenzar, el punto de partida de la interpretación ha de ser la delimitación del texto, objeto de investigación; esto implica definir una dimensión espacial y temporal que implique cierta precisión sobre aquello que será susceptible de comprensión, sin menosprecio de la analogicidad que abre las puertas a múltiples posibilidades delimitativas, tanto en función de la realidad como del intérprete y su potencial cognitivo. En este sentido, la hermenéutica analógica reconoce el punto de partida en el interés del intérprete sobre lo que quiere comprender, más que agentes externos que de forma unívoca determinan porciones investigables de la realidad. Así, la delimitación del objeto es cuestión del interés y del conocimiento previo del intérprete, en superación de las visiones que asumen la neutralidad como garantía de objetividad desconociendo la historicidad del sujeto; plantea Bachelard: "hallar el objeto es 
verdaderamente hallar el sujeto" (citado en Alvarez, p. 239).

Con todo, la apertura analógica metodológicamente define también, una condición de cierre, que evitaría el equivocismo de no plantear límites claros; junto a la validez de los intereses propios de los intérpretes, -intereses ligados a su posicionamiento cognitivo-, el criterio estaría dado en la ubicación del texto a partir de la especificidad de su contexto, tema que no es ajeno a otros enfoques hermenéuticos. Esta contextuación ha de darse en niveles intra, inter y extratextuales, en relación con los planteamientos del texto en sí mismo, los debates en los que interviene y el entorno de la generación que le ha dado origen; ubicar el texto en estos escenarios se convierte entonces en una exigencia cognitivo-interpretativa de todo intérprete; se remite a la comprensión misma del objeto de investigación.

De tal forma, una vez lograda la delimitación del texto, se da paso a su contextuación. Los tres niveles planteados se convierten en vías para adentrarse en la comprensión del mismo. En primer lugar, se hace referencia a la sintaxis, que permite el descubrimiento del significado intratextual -e incluso intertextual, si se tiene en cuenta la sintaxis como fundamento indispensable de la semántica y la pragmática-. En segundo lugar, se involucra el significado del texto mismo, ya no como sentido sino como referencia, esto es, permite llegar al mundo o realidad del texto. En tercer lugar, correspondiente a la pragmática, se logra involucrar en el proceso la injerencia de las personas en la significación y la comunicación; implica la intencionalidad del autor y su inserción en un contexto.

Esto coincide además con tres tipos de verdad que se darían en el texto: una verdad sintáctica, como pura coherencia, que puede ser tanto intratextual (al interior del texto) como intertextual (con otros textos relacionados); una verdad semántica, como correspondencia con la realidad (presente o pasada) o con algún modo posible (futuro o imaginario) a que el texto alude, y una verdad pragmática, como convención entre los intérpretes (e incluso con el autor) acerca de lo que se ha argumentado y persuadido de la interpretación, a pesar de que contenga elementos extratextuales (subjetivos o colectivos). (Beuchot, 2009, p. 21).

Dicho esto el método de la hermenéutica estaría dado por las dimensiones semióticas de sintaxis o implicación, semántica o explicación, y pragmática o aplicación.

Un elemento adicional que se da en la hermenéutica analógica y que conlleva implicaciones metodológicas, es la condición misma de comprensión, ligada a la explicación. Plantea Alvarez: "es el hecho de que sin un mínimo de conocimiento y de comprensión el 
intérprete está impedido para generar explicación alguna, de ahí que ese comprender lleve implícita una condición de explicación" (p. 242). Aparece entonces una concepción de hipótesis en la hermenéutica analógica como el supuesto previo que el intérprete tiene del texto que está buscando comprender y que más allá de ser verificado o rechazado, se constituye en elemento de orientación para la comprensión que se va elaborando; este supuesto es susceptible de cambio conforme se avanza la investigación, en una lógica de descubrimiento y transformación de las hipótesis interpretativas de origen. El proceso avanza desde una elaboración explicativa, en la cual con cierto margen de interpretación, los enunciados hipotéticos son ratificados o modificados. De hecho, las habilidades interpretativas y el trabajo de investigación previo del investigador, juegan un rol crucial, y bajo ese supuesto, la hermenéutica analógica asume la posibilidad de antinomias o paradojas entre dos o más interpretaciones, susceptibles de ser jerarquizadas a partir de criterios de valor proporcional como los márgenes de aceptación de una comunidad o una tradición. Las "verdades" alcanzadas por la hermenéutica analógica estarían dadas por atribución o correspondencia, lo cual puede ser contrastable ya sea con ciertos hechos o con el texto que se está interpretando.

Finalmente, si bien el acto interpretativo de la hermenéutica analógica podría concluir con la elaboración de una explicación, dado el carácter del proyecto el ejercicio será llevado en las tres fases, es decir, hasta el ámbito aplicativo que comporta el carácter pragmático. Planteado en esta perspectiva, todo el proceso implica el siguiente razonamiento: inicialmente, se elige una o varias hipótesis; luego, el intérprete se enfrenta al texto y a partir de él elabora una interpretación, según la cual se le da una intensión o sentido al texto, y más adelante, por extensión o referencia, se lleva a un objeto o situación referido por ese texto.

Opciones metodológicas. Expuestos los referentes metodológicos de la hermenéutica analógica, se plantean las opciones mediante las cuales, se concretaron estos referentes: primero, la consideración de las experiencias de docentes como texto susceptible de interpretación analógica; segundo, la entrevista cualitativa semi-estructurada como técnica de recopilación; tercero, las implicaciones y el protocolo del análisis textual; y, finalmente, el proceso de conformación de la muestra junto a algunos argumentos que apoyan la selección escogida. Lo anterior permitirá la presentación, en el apartado final, de las fases y el diseño específico con los cuales se abordó el proceso investigativo.

La experiencia como texto. Una de las principales apuestas es el reconocimiento del proyecto como una oportunidad de aproximación al ámbito del sentido, que remite a lo profundo del ser humano, lo existencial, y que se traduce en prácticas vitales o experiencias concretas en distintos campos de desempeño social. Ahora bien, a nivel metodológico, se asume la concepción del sentido desde la semiótica que permite una aproximación narrativa en 
la cual las experiencias vitales se convierten en textos susceptibles de análisis e interpretación. El objeto es entonces la búsqueda de un significado analógico de las experiencias, en superación del absolutismo de interpretaciones y sentidos únicos, y asimismo, evitando la dispersión de significados múltiples sin jerarquía ni orden, sino siempre a través de un concepto o razón que les dé cierta unidad.

Como ya se ha planteado, el texto a interpretar analógicamente está constituido por las experiencias de docentes con asignación académica en Educación Religiosa, en las instituciones educativas distritales de Bogotá. Cabe entonces recordar la tradición a la que pertenece el concepto experiencia, que lo ubica entre la fenomenología y la hermenéutica, y lo convierte en heredero de autores que van desde Husserl en relación con el mundo de la vida y la experiencia básica de la conciencia no interpretada, hasta las consideraciones sobre la intersubjetividad de Schütz, pasando por Heidegger quien, desde su ser-ahí y ser-en-el-mundo, ubica el sentido de la experiencia en la comprensión; por Merleau-Ponty quien añade a la mediación del lenguaje, la mediación social en reconocimiento del ropaje cultural y emocional que rodea la experiencia; y por Gadamer quien opta por el concepto de vivencia como objetivación de la experiencia en forma de realidad pensada. De Souza (2010) resalta que Heidegger, Gadamer y Schütz coinciden en el lenguaje del sentido común como principal instrumento de la narrativa de las experiencias (p. 255).

Dicho lo anterior, para el presente proyecto se seguirán las consideraciones de Leonardo Boff (2002) quien se basa en la etimología de la palabra para afirmar:

Ex-peri-encia es la ciencia o el conocimiento (ciencia) que el ser humano adquiere cuando sale de sí mismo (ex) y trata de comprender un objeto por todos los lados (peri). La experiencia no es un conocimiento teórico o libresco, sino que se adquiere en contacto con la realidad, que no se deja penetrar fácilmente y que incluso se opone y resiste al ser humano (p. 12).

La experiencia supone entonces el acceso al conocimiento a través del encuentro con la realidad entendida desde lo vital. De esta manera, por una parte, se logra superar la sensación simple ya que se da una síntesis de percepciones y comprensiones alrededor de lo experimentado, y por otra, se reconoce en el mismo ejercicio la condición humana de estar orientado hacia fuera, expuesto y abierto a; en otras palabras, el reconocimiento del ser humano como existencia y por lo tanto en diálogo con el otro y con el mundo. Por esto último, no es equiparable la experiencia con la vivencia pues se reduciría a una situación psicológica en la subjetividad, negando que en el movimiento de salir de sí al encuentro con el mundo, la persona se lleva a sí misma como lente de comprensión de la realidad; la experiencia contiene entonces un elemento subjetivo y un elemento objetivo, y sólo se estructura en el encuentro y 
modificación que ambos sufren. "Podemos decir que la experiencia es el modo en que interiorizamos la realidad y la forma que tenemos de situarnos en el mundo junto a los demás" y en ese sentido, "posee el carácter de un horizonte", esto es, una perspectiva de la realidad para "descubrir los distintos objetos dentro de ella, nombrarlos y ordenarlos con el rigor de una sistematización" (p. 13).

En coherencia estos postulados, Vivas (2009) presenta la posibilidad de reconocer la experiencia como elemento configurativo del conocimiento, llegando incluso a plantear la experiencia como validación epistemológica del mismo. En esa línea, plantea la relevancia fundamental del lenguaje como expresión de experiencias de sentido, del mundo, y como comunicación de su propia existencia; afirma:

Para que el ser humano tenga algo que decir a través del lenguaje, su "existencia se ha hecho experiencia". [La experiencia] por tanto, hace referencia a situaciones ya vividas, que posteriormente se volverán reflexivas, en cuanto se extienden a un grupo humano o comunidad y de ahí surge una conceptualización o conocimiento (p. 184185).

Aunque se hace la salvedad de que probablemente no todo lo manifestado por el ser humano en su esfuerzo por comunicarse configure una experiencia reflexiva, hay claridad en la percepción de una fuerza vital en el ser que se comunica y en la implicación necesaria de la voluntad de "querer leer y transmitir en otros/as, situaciones que bien pueden ser traducidas como experiencias de vida individual y colectiva" (p. $185)$.

Ahora bien, para el caso del proyecto la recopilación de las experiencias se realizó a través de entrevistas semi-estructuradas sobre las cuales se desarrolló un protocolo de análisis textual; a la explicación de estos aspectos, se dedican los siguientes apartados.

Entrevistas semi-estructuradas. La entrevista es una de las técnicas más comúnmente utilizadas por el paradigma cualitativo de investigación; se caracteriza por recopilar la información a través de una conversación en la que se intenta entender el sentido de lo que el entrevistador dice y no sólo los datos que este plantea. A continuación, se exponen planteamientos en torno a su finalidad y el carácter de la indagación, para llegar a la consideración de las entrevistas semi-estructuradas y el rol del investigador en ellas. 
Si bien, la inmersión en la realidad social que efectúa el investigador con la entrevista cualitativa no es tan profunda como la que se realiza con técnicas como la observación participante, comparten el objetivo de acceder a la perspectiva del sujeto estudiado, en el sentido de comprender sus concepciones subyacentes, interpretaciones, motivaciones y percepciones. La finalidad es entonces la recopilación de la experiencia con un horizonte mayor de comprensión a través del conocimiento y percepciones del entrevistado; de hecho, la dinámica de la entrevista supone un contenido cambiante en relación con la disposición y los planteamientos del entrevistado. En este sentido, la entrevista puede ser considerada un instrumento no neutral pues quienes intervienen en ella producen la realidad de la situación de la misma (Vargas, 2012); así, la dinámica de la misma supone apertura y flexibilidad en aras de que los elementos preestablecidos no coarten la expresión libre de las experiencias por parte de los entrevistados.

Frente al protocolo de preguntas preestablecidos con cierta rigidez propio de las entrevistas estructuradas, las entrevistas semi-estructuradas y no-estructuradas proveen una mayor amplitud de alternativas en el paradigma cualitativo. Las preguntas reflejan ideas que se esperan que circulen a través de las respuestas del entrevistado sin coartar las ideas emergentes y las distintas opciones de abordaje que este pueda asumir. De esta forma, la pregunta más que un instrumento para recoger datos, es un estímulo para suscitar el compartir de la experiencia y la comprensión de la misma.

Las entrevistas entonces se clasifican en relación con su grado de estandarización, esto es, la libertad o coerción que se concede al entrevistador y al entrevistado. El criterio de flexibilidad admite también una graduación; entre la entrevista estructurada -en la que a todos los entrevistados se les hacen las mismas preguntas con la misma formulación y en el mismo orden-, y la entrevista no estructurada -donde el contenido de las preguntas no está establecido y varía en función de cada sujeto-, se sitúa la entrevista semi-estructurada -donde el entrevistador dispone un guión que recoge los temas a tratar con la entrevista-. En ella, el orden en que se abordan los temas y el modo de formular las preguntas se dejan a la libertad del entrevistador de acuerdo con la situación y la disposición del entrevistado; esto es, se realizan las preguntas oportunas en los términos que se estima inconvenientes y se solicitan aclaraciones, énfasis, profundizaciones, de manera que se cree un estilo personal y propio para la conversación. 
Mientras que una entrevista estructurada con cuestionario preestablecido pone al entrevistado dentro de esquemas ya fijados por el investigador, la entrevista semiestructurada busca una comprensión más profunda, a través de la ampliación por parte del entrevistado de sus consideraciones sobre un tema de mayor interés; de tal manera, se pueden saltar preguntas y abordar temas novedosos de acuerdo con las vivencias, intereses y necesidades que puedan manifestarse en la entrevista. La voz imperante debe ser la del entrevistado quien con libertad puede expresar su modo de ver las cosas, motivaciones y pensamientos. Para ello, se distingue entre preguntas primarias -que introducen un nuevo tema o un nuevo interrogante-, y secundarias -que articulan y profundizan el tema inicial-. Asimismo, son importantes los estímulos para animar al entrevistado a profundizar en ciertas cuestiones: repitiendo la pregunta con otras palabras, repitiendo la respuesta o la idea del entrevistado, manifestando expresiones de interés, haciendo pausas, o bien, solicitando explícitamente la profundización en algún tema. Como se planteó, esto favorece la dinámica de la entrevista como interacción entre personas, más que como simple recopilación de datos.

Este carácter de flexibilidad como adaptación a las necesidades y características de los sujetos requieren mayor preparación por parte del investigador tanto para el abordaje de la técnica como para el análisis posterior. En esta perspectiva, el rol del entrevistador es fundamental en términos de propiciar cordialidad, comodidad y tranquilidad en el entrevistado; es decir, permitirle expresar la experiencia de forma espontánea, a través de la escucha paciente, el respeto por las pausas y silencios, y el evitar juicios valorativos y discusión sobre las respuestas. Este favorecimiento de la conversación y el relato del entrevistado, a través de una actitud empática, es un aspecto fundamental para el éxito y legitimidad de la entrevista. Si el objetivo es acceder al mundo de los sentidos, emociones y pensamientos auténticos, el poder generar un ambiente de confianza pese a las limitaciones del tiempo, adquiere relevancia en el reconocimiento de la entrevista como un proceso de interacción social entre dos personas. Teniendo esto en cuenta, el proceso inicial de acercamiento debe encaminarse a romper el halo de desconfianza natural y la inseguridad ante la situación desconocida; para ello, deben expresarse con claridad los objetivos de la entrevista, el porqué de su inclusión en la muestra, y, las razones para abordar algunos aspectos más personales. En el caso particular del proyecto, la similitud de la situación del entrevistador y los 
entrevistados, en la situación laboral, formación profesional, vinculación distrital, supuso una ventaja significativa en el sentido expuesto.

El análisis textual. Recopiladas las experiencias se abre paso la pregunta por el análisis e interpretación de las mismas. Ya Santander (2011) plantea que "no existe la técnica para hacer análisis" (p. 215), en el sentido de que es imposible encontrar un técnica o protocolo único que sea útil y adecuado para todos los estudios. La pertinencia depende de las problemáticas particulares y las motivaciones del investigador, expresadas en la pregunta y el objetivo planteados. Se advierte entonces cómo en las investigaciones cualitativas, pese al reducido número en la muestra, el volumen de información tiende a ser considerable y en el caso del presente proyecto, la utilización de entrevistas semi-estructuradas no escapa a esta lógica. De hecho, las transcripciones generan un volumen importante de texto a ser analizado, ante lo cual es importante remitirse continuamente a lo que se busca en el texto, esto es, el establecimiento de criterios de jerarquización para la organización del material recolectado; por supuesto, estos no pueden ser otros que los referentes teóricos y la pregunta de investigación. En este sentido, el análisis es necesariamente un proceso selectivo en el que no todo puede ser abarcado.

Cuando los datos cualitativos son textos, Fernández (2006), presenta dos formas de asumirlos: como objetos de análisis en sí mismos desde la tradición lingüística, o como una ventana a la experiencia humana desde la tradición sociológica. En el primer caso, se incluyen el análisis narrativo, conversacional, de ejecución y el lingüístico formal. En el segundo caso, se distinguen dos tipos de texto: las palabras o frases generadas por medio de elicitación sistemática, $\mathrm{y}$, los textos libres entre los que se cuentan las narrativas, los discursos y las respuestas a entrevistas no estructuradas, esto es, con preguntas abiertas. Al proyecto ubicarse en el marco del texto como ventana a la experiencia, y haber suscitado una producción de textos libres, se optó por el método de reducción del texto a categorías que se constituirían en criterios de organización del material y en referentes interpretativos.

El orden del proceso es el siguiente: primero, la obtención de la información, en este caso, a través de las entrevistas; segundo, la captura de la información a través de grabación, y su transcripción; tercero, la codificación, a través de la cual se agrupa la información en las categorías que concentran ideas, conceptos o temas similares de 
acuerdo con los intereses investigativos; y cuarto, la integración entendida como el establecimiento de relaciones de las categorías entre sí y con los fundamentos teóricos de investigación. De tal manera, en un comienzo la codificación fragmenta las transcripciones en categorías distintas, y una vez que se encuentran los conceptos y temas individuales, se elabora una explicación integrada, a partir del examen y comparación del material tanto al interior de cada categoría como entre ellas, buscando establecer relaciones.

En esta perspectiva, la codificación se entiende como categorización con el objetivo de encontrar los sentidos dentro de la complejidad de temas y alusiones que se dan en las transcripciones textuales; esto implica encontrar patrones en los datos, etiquetar los temas y desarrollar sistemas de categorías. De hecho, se entienden los códigos como "etiquetas que permiten asignar unidades de significado a la información descriptiva o inferencial compilada durante una investigación. En otras palabras, son recursos mnemónicos utilizados para identificar o marcar los temas específicos en un texto" (p. 4). Se utilizan posteriormente para recuperar y organizar dichos trozos de texto mediante un sistema que los categoriza y permite su rápida identificación y agrupación en relación con la pregunta, hipótesis o interés investigativo. La creación de los códigos tiene como principales fuentes los referentes teóricos que ya expresan categorías relevantes en el marco investigativo, y los textos mismos de donde pueden inferirse de forma inductiva; en ambos casos, los códigos pueden cambiar y desarrollarse a medida que avanza el trabajo, la importancia radica en su definición precisa acorde con la estructura conceptual, esto es, la coherencia y relevancia de cara al logro del objetivo planteado. En ese sentido, los códigos permiten su establecimiento en distintos niveles correspondiente con los niveles de interpretación planteados por la hermenéutica analógica.

El proceso de codificación y categorización implicó varias tareas: en primer lugar, el muestreo, es decir, la identificación del corpus de textos y sus unidades de análisis, que en el presente caso se dio por las entrevistas y los cuatro ejes en que estas giraron; en segundo lugar, la identificación de los temas, donde se tuvieron en cuenta los temas emergentes como los establecidos en los referentes teóricos; en tercer lugar, la construcción del sistema de códigos, en forma de listas y teniendo en cuenta, los tres niveles de la hermenéutica; en cuarto lugar, la marcación de los textos, es decir, la 
asignación de las etiquetas a las unidades de análisis establecidas que permitió su recuperación e indexación. En quinto lugar, la construcción de modelos conceptuales, a partir de las relaciones entre las categorías.

Teniendo en cuenta lo anterior, para el análisis e interpretación de las experiencias a través de las entrevistas, se escogieron las categorías en relación con los temas principales de investigación. Como ya se planteará en la presentación de las fases y diseño, de acuerdo con cada nivel, se señalaron las categorías que tomaron formas de códigos a identificar en los textos, en un proceso de relectura continua con miras en la precisión conceptual; en el nivel implicativo, se asumieron los ejes principales de las entrevistas así como ciertas concepciones subyacentes; en el nivel explicativo, se asumieron los criterios derivados del análisis teórico y las posibilidades analógicas; finalmente, en el nivel aplicativo, se asumieron los elementos relacionados con la reflexión teórica sobre lo antropopedagógico. Los resultados se presentan en perspectiva descriptiva, utilizando en ocasiones, las mismas palabras de los entrevistados; de esta manera, se presentan los razonamientos y las relaciones establecidas, y luego se reproduce un fragmento de las entrevistas como medio de apoyo y explicación.

Conformación de la muestra. Como ya se planteó, la entrevista cualitativa se dirige a sujetos elegidos de acuerdo con determinadas experiencias o categorías sociales, que se constituyen en criterios específicos. En este caso, se esgrimió como criterio básico: docentes de Instituciones Educativas Distritales de Bogotá, con asignación académica en Educación Religiosa Escolar. Ya en el capítulo inicial, se dieron los argumentos a favor del trabajo con docentes, dado el rol fundamental que cumplen en el proceso educativo: la recuperación del conocimiento generado en el espacio escolar, la situación distrital donde buena parte de la asignación en Educación Religiosa no corresponde a docentes formados para ello, la intencionalidad de una construcción intersubjetiva desde la conciencia de las propias prácticas, entre otros.

Dicho lo anterior, se estableció como población para la recopilación de experiencias a través de entrevistas, la totalidad de 20 docentes, uno por cada localidad del Distrito Capital de Bogotá, de acuerdo con su división político administrativa que también corresponde a la organización de la Secretaría de Educación, que plantea en su página web: "cada una de estas localidades presenta una realidad y una dinámica particular" (SED, 2016). En este sentido, teniendo en cuenta el paradigma cualitativo de 
la investigación, la validación de la muestra radica, más que en la representatividad cuantitativa, en la significatividad de la experiencia de los docentes en torno a las categorías centrales; asimismo, los datos diagnósticos que se presentan no buscan una generalización de los resultados al estilo estadístico, sino una ubicación contextual de la situación de donde emerge esta experiencia.

La opción por este tipo de muestra, encuentra en Martínez (2009) varios argumentos a favor: en primer lugar, la intencionalidad de comprender fenómenos humanos que supone la implicación de asumir un saber siempre desde su contexto humano, esto es, sus intereses, valores, disposiciones. En segundo lugar, la consideración del valor de un elemento por sus relaciones y por el estado de otros miembros del sistema; en este sentido, el método de análisis implica tener en cuenta las interacciones que se establecen, en reconocimiento de los sistemas a los que pertenecen los individuos, como estructuras dinámicas que exigen la percepción de otros factores y constituyentes. Sigue en esto, un patrón estructuralista que como se planteó, establece una tensión con el enfoque histórico. Se plantea:

Una misma jugada, por ejemplo, de un futbolista, puede ser genial, puede ser nula y puede ser también fatal para su equipo (autogol); todo depende de la ubicación que tienen en ese momento sus compañeros y sus adversarios. La jugada en sí misma no podría valorarse. Lo que se valora entonces, es el nivel de sintonía de la jugada con todo el equipo, es decir, su acuerdo y entendimiento con los otros miembros (p. 119).

Partiendo de esto, la muestra permite contrastes y comparaciones entre distintas posturas sobre los temas de interés, sin que necesariamente se tienda a la generalización. En esa perspectiva, el reconocimiento de la interacción entre distintos miembros y de la intervención de factores distintos, que hacen que la muestra sea heterogénea, se constituye en una fortaleza más que en una debilidad.

En tercer lugar, se cuestiona la generalización como objetivo esencial de toda investigación, reconociendo el valor que cada vez más han ido adquiriendo las verdades locales; frente a la duda por la generalización a partir de un solo caso o de unos pocos, se afirma: "la generalización es posible porque lo general sólo se da en lo particular" (p. 120).

La lógica que aquí se sigue, según Yin (2003), es la misma lógica que sigue el científico experimental, al pasar de sus resultados experimentales a la teoría; en efecto -dice este 
autor-, el científico experimental no escoge experimentos "representativos"; sencillamente, realiza un experimento, observa el fenómeno, recoge datos y teoriza (p 120-121).

De esta forma, frente a la muestra de una entrevista cerrada, cuantitativa, que se construye con miras en que pueda ser representativa cuantitativamente, esto es, que apunta a la generalización simple, las entrevistas cualitativa semi-estructurada y noestructurada descartan la idea de una población numerosa de entrevistados y atienden a la diversidad de las circunstancias en las que estos pueden encontrarse.

En cuarto lugar, Martínez alude a los principios holográficos que presentan una alternativa al criterio de relación tradicional entre las partes y el todo; de hecho, cada punto del holograma contiene de forma codificada la imagen completa del objeto ( $\mathrm{p}$. 121). Se deriva de esto, la posibilidad de estudiar una comunidad completa a través del conocimiento profundo de algunos de sus miembros, así como, la transferibilidad a partir de la similitud de los contextos. Beuchot (2004) presenta planteamientos similares en sus consideraciones sobre el que denomina un personalismo analógico-icónico. Allí, al exponer la cuestión antropológica asume al ser humano como animal hermenéutico y analógico, pero más aún, expone la idea de la persona como microcosmos es decir, como “análogo e ícono de todo el universo" (p. 9). En esa perspectiva, el ícono es analógico y el ser humano al ser considerado analógico, permite el reconocimiento del rostro del otro como ícono, esto es, como reflejo de la individualidad, su historia e identidad particular, pero al mismo tiempo y sin minusvaloración alguna, como reflejo de la humanidad completa, de todos los seres humanos, de la especie en cuanto tal. Así, la diversidad de la muestra termina apoyada por la opción por lo analógico, cuyo horizonte, más que el de la homogeneidad como fin último, es el reconocimiento de la diferencia bajo el criterio de la proporcionalidad.

Fases y diseño de investigación. Con base en los presupuestos anteriores, el trabajo de elaboración de un marco antropopedagógico vinculante de la formación política al área de Educación Religiosa Escolar se plantea en tres grandes fases correspondientes a los objetivos propuestos y a los niveles interpretativos de la hermenéutica analógica expuestos previamente. Partiendo de una delimitación del texto, que en este caso se planteó como las experiencias de docentes de Educación Religiosa de las Instituciones Educativas Distritales de Bogotá, se asume un proceso 
interpretativo en el que se parte del nivel sintáctico o implicativo, se pasa al nivel semántico o explicativo, para llegar al nivel pragmático o aplicativo.

Así, la etapa inicial, que se denominó 'Fase 0', implicó la caracterización del territorio y la población a nivel descriptivo; se realizó entonces una caracterización de la educación en el marco Distrital, especialmente desde un diagnóstico de las condiciones en las que se desarrolla el espacio escolar de la Educación Religiosa. En ese sentido, se tienen en cuenta datos de los documentos oficiales de la Secretaria de Educación de Bogotá, así como, de encuestas que indagaron por información tanto de las instituciones educativas como de los docentes que conformaron la muestra poblacional. Se organizó la información en tres niveles: Local -teniendo en cuenta la organización política del Distrito en 20 localidades bien definidas-, Institucional y Personal. En el primer nivel, se identificaron los datos generales de las localidades a nivel educativo así como en relación con los docentes nombrados para Educación Religiosa. En el segundo nivel, teniendo en cuenta la jornada de pertenencia del docente, se indagó por la intensidad horaria del área de Educación Religiosa en los distintos niveles, el número de docentes que tienen a cargo la asignación académica, y la representatividad del área en el gobierno escolar. En el tercer nivel, la encuesta giró en torno al área de nombramiento del docente, su formación profesional relacionada o no con el área, y la asignación académica destinada a la misma. A partir de estos rasgos particulares de las instituciones y de los docentes se estableció un perfil general antes del análisis de las experiencias.

Posteriormente, con el fin de identificar el sentido que dan los docentes a la relación Religión-Política y su injerencia en el espacio de Educación Religiosa Escolar, la Fase 1 se centró en la recopilación de las experiencias de los docentes a través de entrevistas semi-estructuradas, que permitieron la circulación de los factores considerados relevantes en torno a cuatro ejes fundamentales: primero, la experiencia de acercamiento a la Educación Religiosa (razones de la asignación, receptividad de los estudiantes, postura de las directivas u otros docentes, lineamientos curriculares, didáctica), segundo, concepciones frente al área de Educación religiosa y su fundamentación (objeto de estudio, papel en el marco educativo, contribución a los objetivos generales de la educación), tercero, relación con la formación política y ciudadanía (relaciones Religión-Política, concepto de política, manifestaciones de lo 
político en las clases de Educación Religiosa, pertinencia de esas manifestaciones), y cuarto, vías pedagógicas (vacíos y necesidades en el área, cambios que introducirían, aportes o hallazgos interesantes). Con base en la sistematización de las entrevistas, junto a los elementos anteriores, se identificaron inicialmente, en el nivel implicativo, los conceptos subyacentes de Ser humano, Religión y Política. De esta manera, finalizada la Fase 1, se establecieron los significados de los textos como sentido, esto es, la formulación de la verdad sintáctica.

La Fase 2 dio paso al análisis de las experiencias de los docentes a partir de los principales debates sobre la relación entre religión y política. Partiendo de los conceptos iniciales subyacentes a estos dos ámbitos -nivel implicativo-, se focalizó en los planteamientos respecto a las relaciones entre lo religioso y lo político, así como en las alusiones a aspectos emergentes en la exploración teórica: diversidad, poder y discurso, libertad y pluridimensionalidad. Simultáneamente a dicho proceso se prestó especial atención a las posibilidades de lectura analógica, a partir de las posturas unívocas, equívocas o mediadores de los docentes, en los ámbitos de referencia. Como resultado de este ejercicio se estableció la correspondencia de los sentidos encontrados en la fase inicial con la realidad y las realidades posibles, expresadas en los planteamientos teóricos sobre la relación Religión-Política; esto es la verdad semántica, en términos de identificación de las relaciones Religión-Política en el espacio de Educación Religiosa y de sus condiciones de posibilidad.

La Fase 3 planteaba la elaboración de criterios antropopedagógicos que permitieran vincular la formación política al espacio de Educación Religiosa a nivel Distrital, esto es, el reto de construir saber escolar en Educación Religiosa a partir de las experiencias pedagógicas de los docentes. En ese sentido, a partir de la aplicación de los elementos que fueron emergiendo en las fases anteriores, se procuró la convención entre los autores/intérpretes. Si en la fase anterior, los elementos emergentes de la matriz teórica entre lo religioso y lo político se constituyeron en filtro y contraste para el análisis, en este caso, fue la reflexión propositiva en torno a lo pedagógico y antropopedagógico aplicado a la verdad semántica, la que permitió la construcción de los criterios constituyentes del marco.

Se han presentado las consideraciones metodológicas que permitieron el abordaje del proyecto, tanto en sus elementos teóricos como en las posibilidades 
empíricas y las formas concretas que se asumieron en términos de técnicas y diseño específico.

\section{Referencias bibliográficas}

Álvarez, A. (2009). El corpus categorial y el método en la hermenéutica analógica para la interpretación de los procesos educativos (tesis de Doctorado en Educación). México: Universidad Pedagógica Nacional.

Beuchot, M. (2004). Antropología filosófica. Hacia un personalismo analógico-icónico. Madrid: Fundación Emmanuel Mounier.

Beuchot, M. (2008). Breve exposición de la hermenéutica analógica. Revista Teología 45 (97), 491-502.

Beuchot, M. (2009). Tratado de hermenéutica analógica. Hacia un nuevo modelo de Interpretación. México: UNAM-Ítaca.

Beuchot, M. (2010). Hermenéutica Analógica y Religión. Theologica Xaveriana 60 (169), 2545.

Beuchot, M. (2012). Hermenéutica Analógica y Política. Anacronismo e Irrupción 2 (3) 181193.

Boff, L. (2002). Experienciar a Dios. Petrópolis: (s. e.).

De Souza, M. (2010). Los conceptos estructurantes de la investigación cualitativa. Salud colectiva 6, 3 (pp. 251-261).

Fernández, L. (2006). ¿Cómo analizar datos cualitativos? Butlletí LaRecerca 7, 1-13.

Martínez, M. (2009). Nuevos paradigmas en la investigación. Caracas: Alfa.

Santander, P. (2011). Por qué y cómo hacer análisis del discurso. Cinta de Moebio, Revista de Epistemología de Ciencias Sociales 41, 201-224.

Vargas, I. (2012). La entrevista en la investigación cualitativa: nuevas tendencias y retos. Revista Calidad en la Educación Superior 3, (1) 119-139.

Vivas, M. (2009). La experiencia como validación epistemológica del conocimiento en general y en particular en sujetos específicos. Franciscanum, revista de las ciencias del espíritu 51, (151) 181-206.

Recebido: $18 / 02 / 2016$

Received: 02/18/2016

Aprovado: $30 / 05 / 2016$

Approved: 05/30/2016 